\title{
Comprehensive physical models and simulation package for plasma/material interactions during plasma instabilities ${ }^{1}$
}

\author{
A. Hassanein ${ }^{\text {a,* }}$, I. Konkashbaev ${ }^{\text {b }}$ \\ a Argonne National Laboratory, Argonne, IL 60439, USA \\ b Troitsk Institute for Innovation and Fusion Research, Troitsk, Russian Federation
}

Received 18 May 1998; accepted 28 January 1999

\begin{abstract}
Damage to plasma-facing components (PFCs) from plasma instabilities remains a major obstacle to a successful tokamak concept. The extent of the damage depends on the detailed physics of the disrupting plasma, as well as on the physics of plasma-material interactions. A comprehensive computer package called High Energy Interaction with General Heterogeneous Target Systems (HEIGHTS) has been developed and consists of several integrated computer models that follow the beginning of a plasma disruption at the scrape-off layer (SOL) through the transport of the eroded debris and splashed target materials to nearby locations as a result of the deposited energy. The package can study, for the first time, plasma-turbulent behavior in the SOL and predict the plasma parameters and conditions at the divertor plate. Full two-dimensional (2-D) comprehensive radiation magnetohydrodynamic (MHD) models are coupled with target thermodynamics and liquid hydrodynamics to evaluate the integrated response of plasma-facing materials. Factors that influence the lifetime of plasma-facing and nearby components, such as loss of vapor cloud confinement and vapor removal due to MHD effects, damage to nearby components due to intense vapor radiation, melt splashing, and brittle destruction of target materials, are also modeled and discussed. (C) 1999 Elsevier Science B.V. All rights reserved.
\end{abstract}

\section{Introduction}

Damage to plasma-facing and nearby components as a result of various plasma instabilities remains a major obstacle to a successful tokamak concept. The extent of the damage depends on the detailed physics of the disrupting plasma as well as on the physics of plasmamaterial interactions. The high energy deposited in short periods on plasma-facing materials (PFMs) during disruptions can cause severe erosion, plasma contamination, and structural damage of these components. Erosion can take various forms such as surface vaporization, spallation, and liquid ejection of metallic mate-

\footnotetext{
${ }^{*}$ Corresponding author. Tel.: +1-630 252 5289; fax: +1-630 252 5287; e-mail: hassanein@anl.gov

${ }^{1}$ Paper presented at the 13th International Conference on Plasma Surface Interactions in Controlled Fusion Devices, San Diego, CA May 18-22, 1998.
}

rials. The eroded and splashed materials will then be transported and redeposited on various plasma-facing and other nearby components, possibly leading to serious plasma contamination of subsequent plasma operations.

A comprehensive computer simulation package called High Energy Interaction with General Heterogeneous Target Systems (HEIGHTS) has been developed to study in detail the various effects of sudden high-energy deposition of different sources on target materials. Currently the package does not include models to evaluate the structural deformation and damage from electromagnetic forces evolving during the current quench phase of a plasma disruption. The developed package consists of several integrated models that follow the beginning of a plasma disruption at the scrape-off layer (SOL) through the transport of the eroded debris and splashed target materials as a result of the deposited energy. The developed models explain, for the first time, the plasma turbulent behavior in the SOL and predict 
the plasma parameters and conditions at the divertor plate. To evaluate the magnitude of various damage to plasma-facing and nearby components caused by plasma instabilities, full 2-D comprehensive radiation hydrodynamic models have been developed by using advanced numerical techniques such as Particle-in-Cell (PIC) and Ray Tracing methods. These models with such advanced numerical methods are needed for a realistic analysis of disruption conditions and resulting consequences. Detailed physical models of plasma/solid-liquid/vapor interaction in a strong oblique magnetic field have also been developed, in a fully self-consistent 2-D model that is coupled with radiation hydrodynamic models. Factors that influence the lifetime of plasmafacing components such as loss of vapor cloud confinement and vapor removal due to magnetohydrodynamic (MHD) instabilities, damage to nearby components due to intense vapor-radiation, melt splashing, and brittle destruction/explosive erosion of target materials, are also modeled and addressed. Ongoing benchmarking of various models with available data from both tokamak and laboratory experiments is being actively pursued. The work presented in this study is intended, however, to only briefly describe the HEIGHTS package, emphasizing newer capabilities and summarizing some of the main results that are important in evaluating overall disruption damage and lifetime of plasma-facing components (PFCs) in reactor environment.

\section{Brief description of HEIGHTS package}

The high energy deposited on reactor walls during loss of plasma confinement can cause significant surface damage, structural failure, and plasma contamination of subsequent operations. Surface damage includes high erosion losses due to surface vaporization, spallation, and melt-layer splatter. Bulk damage effects include large temperature increases in structural materials and at the interface between surface coatings and structural materials. These large temperature increases cause high thermal stresses, possible structure melting, and material fatigue and failure. Other bulk effects of some plasma instabilities, particularly those of longer duration such as vertical displacement events (VDEs) or those with deeper deposited energy such as runaway electrons, can cause high heat flux levels at the coolant channels; this may cause burnout of these tubes and result in significant downtimes for repair and maintenance [1]. In addition to these effects, the transport and redeposition of the eroded surface materials from vaporization, melt-layer splashing, and macroscopic particle emission to various locations on plasma-facing and nearby components are of major concern for safety, frequent plasma contamination, and prolonged suc- cessful plasma operations following plasma instability events.

Three key factors significantly influence the overall response and erosion of a PFC as a result of the intense deposited energy during plasma instabilities. These are: (1) characteristics of particle-energy flow (i.e., particle type, kinetic energy, energy content, deposition time, and location) from the SOL to the divertor plate; (2) characteristics of the vapor cloud that developed from the initial phase of energy deposition on target materials and its turbulent hydrodynamics, and (3) the generatedphoton radiation and transport in the vapor cloud and nearby regions. The characteristics of particle-energy flow from the core plasma to the SOL during plasma instability events are being studied with the recently developed SOLAS model in which an analytical solution is derived for plasma particle distribution functions in the SOL by solving the Fokker-Planck equation [2]. The dynamics of target thermal evolution, surface erosion due to vaporization, vapor cloud formation and shielding effects, MHD expansion, vapor turbulent instabilities and loss of confinement are studied using the comprehensive A*THERMAL-S code [3-5].

Most of the incident plasma kinetic energy during a disruption, however, is quickly transformed into photon radiation if the vapor cloud is well confined by the magnetic field. The resulting photon radiation from the continuous plasma heating of the vapor cloud and the transport of the emitted radiation are very important and complicated problems. For such analysis, the PhD and SUPERATOM codes are used and are also coupled to the A*THERMAL-S code. The PhD code calculates detailed deposition of the emitted photon radiation from the vapor cloud to nearby components. The SUPERATOM code calculates the atomic physics data of different target materials in order to calculate the resulting radiation. The behavior and erosion of the free metallic surface of a liquid layer subject to various internal and external forces during the disruption are studied with the SPLASH code [6-8]. In addition, the SPLASH code calculates the explosive erosion and the characteristics of brittle destruction erosion of carbon-based materials (CBMs). Redeposition of the eroded debris and splattered materials is analyzed with the DRDEP code. Tritium behavior and containment in the generated dust and eroded debris of PFMs, as a result of various plasma instabilities, are being analyzed and evaluated with the TRICS and TRAP codes. Details and the models and initial results of these codes are given elsewhere [9]. Detailed particle transport and range, energy depostion, and sputtering erosion are analyzed with the 3-D Monte-Carlo ITMC code, described elsewhere [10]. Fig. 1 schematically describes the complete HEIGHTS simulation package used in the analysis of a beam-ontarget interaction. 


\section{Simulation Package for High Energy Interaction with General Heterogeneous Target Systems}

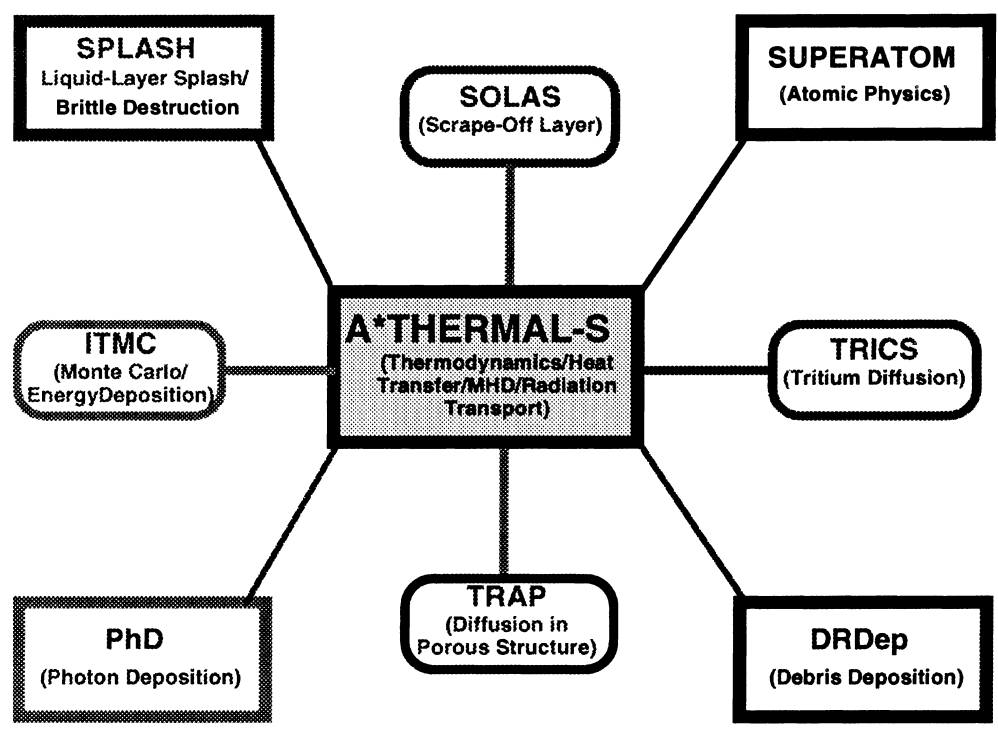

Fig. 1. Schematic description of the HEIGHTS simulation package.

\subsection{Plasma behavior in the scrape-off layer}

The steady operation of a plasma device is largely determined by the boundary conditions at the divertor or the limiter plates. The divertor plate, for example, in future fusion power machines is a key component in removing the particle and heat fluxes. During various plasma instability events, the loss of confinement will cause the majority of the core particle flux to arrive at the SOL with a relatively high temperature $\left(T \approx T_{0} \approx 10\right.$ $20 \mathrm{keV})$, where $T_{0}$ is the core plasma temperature prior to a disruption. This is in contrast to normal operation in which the escaping particles from the core plasma to a collisional SOL have a relatively lower temperature, $T<1 \mathrm{keV}$. Because of the high temperature of the escaping particles during plasma instability events, the SOL plasma becomes collisionless and requires different treatment than that during normal operation.

The initial response of target materials to a plasma disruption depends on the incident energy spectra of plasma ions and electrons. Recent calculations and experimental results from various plasma gun and electron beam devices show significant differences in target thermal evolution, in dynamics of the vapor cloud and its radiative properties, and in erosion rates. The inci- dent energy spectra, however, are determined by the physical processes occurring in the SOL such as mechanisms of particle escape and magnitude of the sheath potential formed between the SOL plasma and the divertor walls. Therefore, to accurately evaluate the erosion and lifetime of PFMs, a complete understanding of the disrupting plasma behavior in the SOL is necessary.

One of the major differences found between the SOL during normal operation and during a disruption is that the disrupting high-temperature plasma from the core to the SOL is a collisionless plasma. This will have the initial effect of a much lower electron density in the SOL than of ion density. For charge neutralization, however, another electron population with longer lifetime is needed. These electrons will arise from the negative sheath potential between the SOL and the walls. Such a potential well, therefore, will act as an electrostatic trap for part of the electron population. In the recently developed SOLAS model, the characteristics of particleenergy flow from core plasma to SOL during instability events are calculated and then used as an initial condition to evaluate disruption effects on divertor components. One of the main results of the SOLAS model is that the electron distribution function in the SOL consists of three different populations: Maxwellian part, 
non-Maxwellian part, and monoenergetic [2]. The energy distribution of these populations are determined by the potential well established between the SOL and the walls. This potential is found to be much lower, by one order of magnitude, than in the case of a collisional SOL existing during normal operation. The calculated electron and ion energy spectra are then used to evaluate the thermal evolution of target materials during the early phases of a disruption event.

\subsection{Two-dimensional MHD modeling of target debris}

The sudden formation of a vapor cloud above the target surface from the deposited plasma energy can significantly reduce, if well confined, the net energy flux to the original disruption location, thus substantially reducing vaporization losses by orders of magnitude [1]. Detailed physics of plasma/solid-liquid/vapor interactions in a strong and oblique magnetic field have been developed and implemented in the comprehensive selfconsistent A*THERMAL-S code. Such detailed treatment of the MHD and photon radiation transport in the vapor cloud region is proved to be very important in determining the net erosion thickness resulting from surface vaporization [7].

The vapor cloud plasma in a tokamak environment may also be subject to MHD instabilities, similar to those in the reactor main plasma, and therefore loss of vapor confinement and shielding away from the incoming main plasma particles during the disruption. Initially, the cold vapor plasma with low conductivity near the target surface diffuses freely across magnetic field lines in the direction normal to the surface. This expanding vapor plasma is heated directly, first by the disrupting mainplasma particles and then by electron heat conduction and by photon radiation generated inside the outermost vapor regions. As the cold vapor becomes ionized, it will then turn to follow the initial direction of the oblique magnetic field lines. The magnetic field lines are initially assumed to be frozen into the surface of the liquid metal layer because of the liquid's high conductivity. As more vapor is emitted from the surface, the expanding dense and cold vapor will sweep and distort the oblique magnetic field lines. Near the upper vapor boundary, the magnetic field lines become almost parallel to the vapor surface. Such a situation of distorted magnetic field distribution results in a flute-type MHD instability in the vapor plasma [3]. As a consequence of the loss of vapor confinement, the turbulent diffusing hot vapor will then deposit its energy on nearby components, causing more erosion. The overall net erosion rate and resulting damage will then depend on the disrupting plasma parameters, the size of disruption spot, design configuration, and the type of PFM.

Therefore, for more accurate assessment of disruption damage, a full 2-D MHD analysis of target debris with realistic plasma facing and nearby components geometry is developed. The 2-D version of the A*THERMAL-S code now includes two new significant additions in treating both vapor plasma MHDs and radiation transport. The 2-D MHD part of the code uses the particle-in-cell/cloud (PIC) approach to describe the dynamics of vapor debris in two coordinates: perpendicular to the target surface and along the (poloidal) surface. Magnetic field lines are assumed to have an angle $\alpha$ with the surface. Therefore, two components of the field lines are taken into account. The Lagrangian method is more popular and more suitable for 1-D MHD problems. For 2-D problems, Lagrangian method results, however, in strong cell distortion and large numerical instabilities, particularly for strong nonhomogenous problems. Pure Eulerian methods also result in more numerical instabilities and are not suitable for plasma distributions with steep density profiles. The PIC method uses a nonstationary Eulerian 2-D grid for calculations of vapor plasma parameters such as vapor density, pressure, and temperature, while the particles are used to determine fluid parameters such as mass, velocity, and energy. This requires rezoning of the hydrodynamic grids at every time-step according to the displaced particle positions. This method has the advantage of being more accurate and of avoiding nonphysical oscillation of vapor parameters that occurs when using a stationary grid, particularly in the case of a free boundary problem. As more vapor debris leaves the surface due to further heating of the target, more Eulerian cells are generated with time.

Radiation transport in vapor plasma and photon deposition to surrounding locations nearby the exposed target area are very important and complicated problems. The incident plasma kinetic energy during disruptions is quickly transformed into photon radiation by the vapor cloud. Therefore, an accurate method to account for radiation transport and deposition is essential in evaluating the overall disruption damage. The 2-D radiation transport portion is similar to what is known as the "escaping probability" method, which uses ray approximation techniques for photon propagation. Photon optical thicknesses vary significantly in the developed vapor plasma because the vapor plasma contains regions of very cold and dense plasma near the target surface and very hot and less dense plasma regions where the disrupting plasma ions and electrons deposit their energy. This transport method, unlike the diffusion approximation, forward-reverse, and other methods, is valid for multidimensional problems with any optical thickness ranging from transparent media up to plasma conditions that are in local thermodynamic equilibrium (LTE).

The developed method in this work takes advantage of the spherical/cylindrical wave nature of photon propagation. Therefore, two types of calculational grids 
are used in the 2-D MHD model. The rectangular grid is more appropriate for the solution of hydrodynamic system of equations, while the cylindrical grid is more suitable for radiation propagation. Fig. 2 schematically illustrates the relationship between the rectangular hydrodynamic cells and the cylindrical cell used to calculate the deposited radiation energy from each evolving hydrodynamic cell. The number of cylindrical cells is defined by the number of chosen rays (sectors) and the number of radial sectors. Each cylindrical cell may contain several hydrodynamic cells. This method allows us to calculate photon-deposited energy at any nearby location from each single radiative hydrodynamic cell. Although this method requires more calculational steps than other techniques, e.g., the diffusion approximation methods, it is far more accurate and more numerically stable for a wide range of vapor plasma parameters and conditions.

To demonstrate a few capabilities of this 2-D package, two cases are studied. An unexpected experimental result from plasma guns and other simulation devices is that the spatial distribution of target-eroded material does not depend on the incident beam profile $[11,12]$. Fig. 3 shows the relative radiation power from a carbon vapor cloud for two different incident beam profiles: a rectangular (homogeneous) shape and a half-Gaussian (nonhomogeneous) profile that may be expected during a turbulent loss of core plasma confinement. It can be seen that the developed spatial distribution of the evolving vapor cloud above the exposed surface does resemble the shape of the incident beam profile. However, the spatial distribution of the radiated power to the target surface, and thus of the eroded material, is independent of beam profile, as shown in Fig. 3, and extends beyond the size of the incident beam; this also is in good

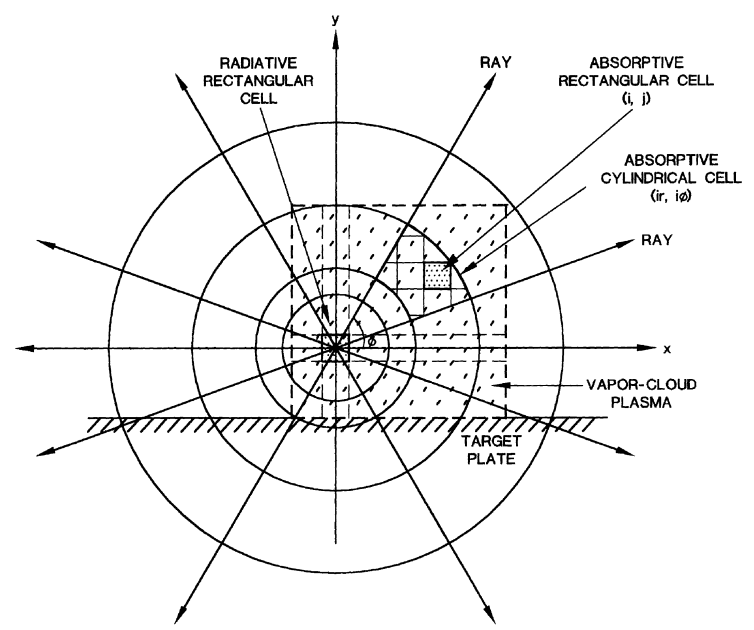

Fig. 2. Schematic illustration of rectangular hydrodynamic and cylindrical radiation cells used in PIC and ray tracing methods.

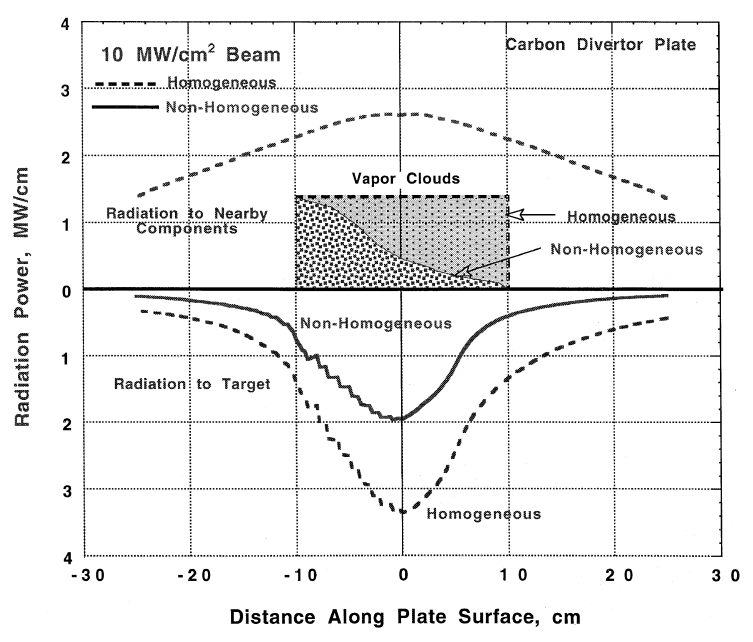

Fig. 3. Radiation power emitted from carbon-vapor plasma for two different incident beam profiles.

agreement with various experimental results $[11,13]$. This can be explained as follows. The quickly developed vapor cloud is heated by the energy of beam and then starts to reradiate the absorbed plasma energy. For a typical vapor plasma developed during a tokamak disruption, the temperature of the vapor ranges from $T \approx 1$ $\mathrm{eV}$ close to target surface and up to $T<100 \mathrm{eV}$ near the outermost vapor regions where the disrupting plasma deposits its energy. However, most radiation is only emitted from a certain region of the vapor plasma near the target surface with lower vapor temperatures $(T \approx 10 \mathrm{eV})$. Most radiation power is absorbed within the vapor cloud or escape elsewhere and therefore cannot reach the target surface. Because this thin strip of the highly radiating zone is close to the surface of the target and because of the geometrical effects, the net power profile (and erosion profile) to the surface becomes independent of the incident beam shape and extends beyond the size of the incoming beam. However, the magnitude of the power to the surface, and therefore also the erosion rate, does depend on the total content of the incident beam energy, as shown in Fig. 3.

A second important finding is that radiation power to the target surface (and therefore, vapor cloud hydrodynamic evolution and target erosion) depends strongly on the accuracy of the radiation transport calculations. Total radiation power is usually composed of both continuum and line radiation. The continuum radiation spectra in a typical vapor plasma generally have a longer path length (vapor plasma is more optically thin) than that for line radiation spectra during a disruption. Therefore, the number of multigroup energy spectra, $K$, can significantly affect the total emitted radiation and the radiated power to surface. Fig. 4 shows the normalized (to the value of $K=1000$ ) radiated power to the surface and the total escaped radiation from a stationary 


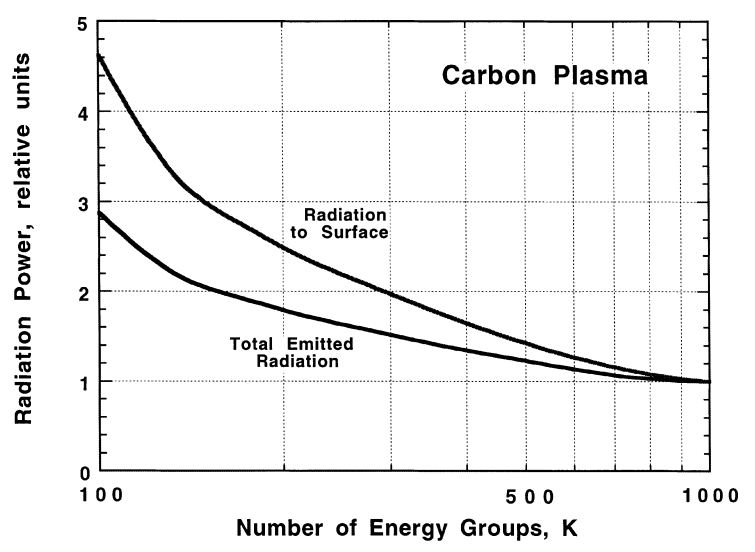

Fig. 4. Dependence of emitted radiation power on number of multigroup energy spectra.

carbon plasma as a function of the number of continuum energy groups used in the calculation. If the number of energy multigroups is less than 1000 , the radiated power and therefore the damage to PFCs and nearby components can be significantly overestimated (by a factor of three or more). This calculation was performed for a carbon plasma with fixed temperature, density, and pressure distribution obtained from a real beam-ontarget calculations mentioned above. Line radiation (which can be as high as $70-80 \%$ of total radiation) is radiated mainly by the outermost high-temperature regions of the vapor plasma where, in this case, the vapor temperature $T<100 \mathrm{eV}$, depending on disruption energy density and deposition time. Most line radiation is due to He-like ions in which each resonance line can radiate up to several MW of power. Regions of line radiation for Li-like ions are usually optically thick in the vapor-like plasma, and their contribution to the total escaping radiation to target surface is small.

The PhD code is designed to calculate detail timedependent photon energy deposition on the target surface and on any nearby components from each radiating hydrodynamic cell of the vapor plasma, due to both continuum spectra and each line radiation of every-single level population in each existing ionization state. Fig. 5 schematically illustrates the geometry used in the $\mathrm{PhD}$ code to calculate photon energy deposition. A nearby component is described by a radial distance, $R_{\mathrm{c}}$, from the center of the disruption location on the divertor plate and by a solid angle, $\Phi$, as shown in Fig. 5 . The intense photon radiation to nearby components in a closed-design configuration can cause more erosion damage than that at the original disruption location [14]. This can occur because of less vapor shielding from the secondary vapor cloud of the nearby components and the longer mean free path of the photon radiation in this optically thin vapor cloud.

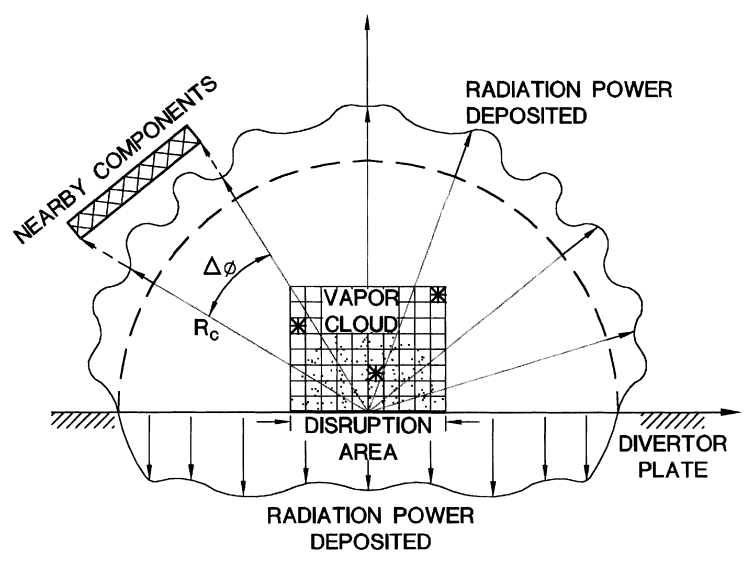

Fig. 5. Geometry used in $\mathrm{PhD}$ code to describe and evaluate response of PFCs to disruption on divertor plate.

\subsection{Macroscopic erosion of PFCs (SPLASH code)}

Fig. 6 shows a typical time evolution of a tungsten surface temperature, melt-layer thickness, and vaporization losses during a disruption for an incident plasma energy of $10 \mathrm{MJ} / \mathrm{m}^{2}$ deposited in a disruption time of $1 \mathrm{~ms}$ as predicted by the A*THERMAL-S code. An initial magnetic field strength of $5 \mathrm{~T}$ with an incident angle of $2^{\circ}$ is used in this analysis. The sharp initial rise in surface temperature is due to the direct energy deposition of incident plasma particles at the material's surface. The subsequent decrease in the surface temperature was caused by the shielding effect of the eroded material accumulated above the target surface. The subsequent behavior of the target behavior is mainly determined by the energy flux from the emitted photon radiation in the vapor cloud, as discussed above, and by vapor-electron heat conduction. As more vapor accumulates above the surface, the vapor becomes more

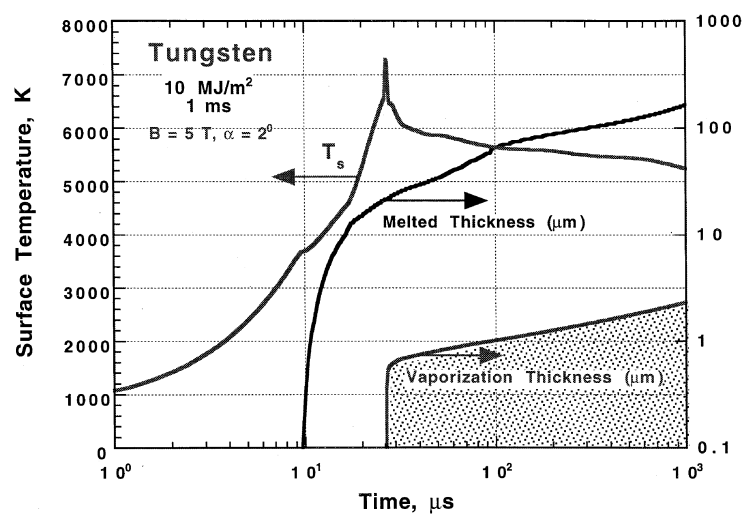

Fig. 6. Time evolution of tungsten surface temperature, melt layer, and eroded thickness following plasma disruption. 
opaque to photon radiation and therefore less energy is transmitted to the target surface.

The resulting melt-layer thickness of a metallic PFC is usually one to two orders of magnitude higher than the surface vaporization losses, as shown in Fig. 6. Therefore, the dynamic response of a liquid-metal layer exposed to various forces during the course of disruption is a serious concern. Models to study detailed macroscopic erosion of metallic materials during an intense deposition of energy were developed, enhanced, and implemented in the SPLASH code. Several mechanisms are found to cause melt-layer loss and splatter during a disruption [7]. These include melt splashing from boiling of gas bubbles, splashing due to absorption of plasma momentum, erosion due to hydrodynamic instabilities developed in the liquid layer from the tangential and perpendicular forces acting on the surface of the liquid, and erosion due to runoff of melt layers over the structure.

Melt-layer erosion generally depends on two main parameters: net power flux reaching the liquid surface, and exposure time [15]. The net power flux to the surface in a typical disruption is calculated to be $\approx 0.5-1.0 \mathrm{MW} /$ $\mathrm{cm}^{2}$, with slight dependence on initial power flux and target material. For a beryllium PFM and typical tokamak disruption conditions of a net power flux to the surface of $300-600 \mathrm{~kW} / \mathrm{cm}^{2}$ and a disruption time of $1 \mathrm{~ms}$, the predicted erosion thickness is about $200-300$ $\mu \mathrm{m}$. A sacrificial beryllium coating thickness of $\approx 5 \mathrm{~mm}$, therefore, will last only for about 15-25 disruptions! This is significantly less than the expected total number of disruptions of about several hundred during the reactor lifetime. Longer disruption times will further reduce disruption lifetime.

Nonmelting materials such as graphite and CBMs have also shown large erosion losses significantly exceeding that from surface vaporization. This phenomenon has been observed in several different disruption simulation facilities such as electron beams [16,17], laser [18], and plasma gun and other devices [11,19]. Models to evaluate erosion behavior and lifetime of CBMs of plasma-facing and nearby components due to brittle destruction during plasma instabilities were also developed and implemented in the SPLASH code.

The macroscopic erosion of CBMs depends on three main parameters: net power flux to the surface, exposure time, and a required threshold energy for brittle destruction [15]. The required energy for brittle destruction is critical in determining the net erosion rate of CBMs and is currently estimated from disruption simulation experiments. From these experiments, an energy for brittle destruction of a graphite similar to the MPG-9 graphite is estimated to be $\approx 10 \mathrm{~kJ} / \mathrm{g}$ or $20 \mathrm{~kJ} / \mathrm{cm}^{3}$. Therefore, for a net power flux to the material surface during the disruption of about $300 \mathrm{~kW} / \mathrm{cm}^{2}$, the depos- ited energy for a time of $1 \mathrm{~ms}$ is $\approx 0.3 \mathrm{~kJ} / \mathrm{cm}^{2}$, which results in a net erosion of $\approx 150 \mu \mathrm{m}$ per disruption. This value is much higher than that predicted from pure surface vaporization of $\approx 10 \mu \mathrm{m}$ per disruption for CBMs [4]. A sacrificial coating/tile thickness about $1 \mathrm{~cm}$ thick would last less than 70 disruptions. Again, this is far less than the current expectations of several hundred disruptions during the reactor lifetime. Longer disruption times can also significantly reduce disruption lifetime. Therefore, more relevant experimental data and additional detailed modeling are needed to evaluate the erosion of CBMs.

\section{Conclusions}

A comprehensive computer package called High Energy Interaction with General Heterogeneous Target Systems (HEIGHTS) has been developed and consists of several integrated computer models that follow the beginning of a plasma disruption at the scrape-off layer (SOL) through the transport of the eroded debris and splashed target materials to nearby locations as a result of the intense deposited energy. The package can study plasma-turbulent behavior in the SOL and can predict the plasma parameters and conditions at the divertor plate. Full 2-D comprehensive radiation magnetohydrodynamic models are coupled with target thermodynamics and liquid-layer hydrodynamics to evaluate the integrated response of plasma-facing materials. Vaporproduced plasma and its confinement are important in further reducing disruption damage to the divertor plate and adjacent components. Photon radiation emitted from the vapor cloud, as well as the turbulent diffusing vapor, can also cause significant damage to nearby components. Both melt-layer splashing of metallic components and brittle destruction of carbon-based materials are serious erosion mechanisms during various plasma instabilities and require further investigation. More simulation experiments that resemble reactor conditions are required before a conclusion is made on selection of plasma-facing materials. The frequency of plasma instabilities in future tokamak machines, however, must be sharply reduced to only a few disruptions during the entire reactor lifetime. Moreover, the effects of redeposited debris from the eroded and splattered materials on plasma contamination and on subsequent reactor operations need to be studied in detail.

\section{Acknowledgements}

The work is supported by the US Department of Energy, Office of Fusion Energy. 


\section{References}

[1] A. Hassanein, Fusion Technol. 30 (1996) 713.

[2] A. Hassanein, I. Konkashbaev, Physics of collisionless scrape-off-layer plasma during normal and off-normal fokamak operating conditions, Argonne National Laboratory Report, ANL/FPP/TM-296, March (1999).

[3] A. Hassanein, I. Konkashbaev, Plasma Dev. Operat. 5 (1998) 297.

[4] A. Hassanein, I. Konkashbaev, J. Nucl. Mater. 233-237 (1996) 713.

[5] A. Hassanein, Fusion Technol. 26 (1994) 532.

[6] A. Hassanein, Fusion Technol. 15 (1989) 513.

[7] A. Hassanein, I. Konkashbaev, Suppl. J. Nucl. Fusion 5 (1994) 193.

[8] A. Hassanein, G. Federici, I. Konkashbaev, et al., Fusion Eng. Des. 39\&40 (1998) 201.
[9] A. Hassanein, B. Wiechers, I. Konkashbaev, J. Nucl. Mater. 258-263 (1998) 295.

[10] A. Hassanein, D. Smith, Nucl. Instrum. and Meth. B 13 (1986) 225.

[11] N.I. Arkhipov et al., J. Nucl. Mater. 233 (1996) 767.

[12] T. Tanabe et al., J. Nucl. Mater. 241-243 (1997) 1164.

[13] A. Hassanein et al., J. Nucl. Mater. 241-243 (1997) 288.

[14] A. Hassanein, I. Konkashbaev, in: C. Varandas, F. Serra (Eds.), Fusion Technology, 1996, p. 379.

[15] A. Hassanein, I. Konkashbaev, L. Nikandrov, Theory and physical models of material erosion during plasma interactions with target surfaces, J. Nucl. Mater., submitted.

[16] V. Engelko et al., J. Nucl. Mater. 220-222 (1995) 1071.

[17] J. Linke et al., in: B. Keen, M. Huguet, R. Hemsworth (Eds.), Fusion Technol., 1991, p. 428.

[18] J. Van der Laan, J. Nucl. Mater. 162-164 (1989) 964.

[19] A.V. Burdakov et al., J. Nucl. Mater. 233-237 (1996) 697. 